\title{
The impact of psychosocial factors on claims outcomes in the group income protection market: findings from a prospective pilot study
}

Carly Van Den Akker

Swiss Re Life \& Health, Sydney NSW 2000, Australia. Carly_VanDenAkker@swissre.com

Research suggests that psychosocial factors predict recovery better than biomedical factors alone in the normal population. However, no studies have been carried out in the disability income insurance setting, where the availability of financial cover is usually perceived as the main detractor of recovery and return to work.

Swiss Re's clinical claims team in collaboration with the Research \& Development team, conducted a 3 year prospective study to evaluate the role of psychosocial factors in recovery within a group income protection sample. Predictors of return to work were: beliefs about recovery and work, work ethic, coping, and self-efficacy. Interestingly, medical diagnosis alone and treatment did not predict recovery and return to work in this sample.

Our objective was to conduct research to gain an understanding of psychosocial predictors in the insurance setting, and additionally to develop specific claims management strategies for our claims assessors to manage their claims more effectively through exploring the psychosocial barriers influencing on a claimant's absence, and providing adequate support at the right time, in a cost-effective manner. 